\title{
L'HOMME L'Homme
}

Revue française d'anthropologie

157 | janvier-mars 2001

Représentations et temporalités

\section{Thomas Spear \& Isaria N. Kinambo, eds., East African Expressions of Christianity}

Oxford, James Currey - Dar es-Salaam, Mkuki na Nyota - Nairobi, East African Educational Publishers (EAEP) - Athens (Ohio), Ohio University Press, 1999, XII + 340 p., bibl., index, ill, carte (" Eastern African Studies »)

Catherine Baroin

\section{(2) OpenEdition}

Journals

Édition électronique

URL : http://journals.openedition.org//homme/5819

DOI : 10.4000//homme.5819

ISSN : 1953-8103

Éditeur

Éditions de l'EHESS

Édition imprimée

Date de publication : 1 janvier 2001

Pagination : 298-300

ISBN : 2-7132-1357-6

ISSN : 0439-4216

Référence électronique

Catherine Baroin, «Thomas Spear \& Isaria N. Kinambo, eds., East African Expressions of Christianity », L'Homme [En ligne], 157 | janvier-mars 2001, mis en ligne le 23 mai 2007, consulté le 24 septembre 2020. URL : http://journals.openedition.org//homme/5819; DOI : https://doi.org/10.4000//homme. 5819

Ce document a été généré automatiquement le 24 septembre 2020.

(c) École des hautes études en sciences sociales 


\section{Thomas Spear \& Isaria N. Kinambo, eds., East African Expressions of Christianity}

Oxford, James Currey - Dar es-Salaam, Mkuki na Nyota - Nairobi, East African Educational Publishers (EAEP) - Athens (Ohio), Ohio University Press, 1999, XII + 340 p., bibl., index, ill, carte (« Eastern African Studies »)

\section{Catherine Baroin}

1 LA RECHERCHE en Afrique de l'Est fait preuve d'un grand dynamisme, sous l'impulsion d'un petit groupe d'historiens de langue anglaise dont on retrouve les noms d'un ouvrage à l'autre dans le catalogue de James Currey. Le dernier-né, édité par Thomas Spear et Isaria Kimambo, en est un signe de plus. Il est le fruit d'une recherche à laquelle ont collaboré l'Université du Wisconsin et celle de Dar es-Salaam. Douze de ses quinze auteurs, ont en effet été formés ou ont enseigné dans l'une ou l'autre de ces deux universités. L'ouvrage s'organise autour de trois idées fortes : rendre compte de l'extraordinaire foisonnement du christianisme en Afrique de l'Est, faire ressortir le point de vue des Africains, et donner un aperçu de la très grande diversité des situations d'un endroit à l'autre. Sur ces trois points, la démonstration est convaincante.

2 La vivacité du christianisme africain n'est certes pas particulière à l'Afrique de l'Est. Elle touche l'ensemble du continent, a attiré l'attention de nombreux chercheurs et a fait l'objet de multiples publications. Son ampleur est telle que l'on pourrait bien le qualifier, comme le suggère Thomas Spear, de «legs le plus important du colonialisme»(p.4). Cet ouvrage apporte sur le sujet des illustrations saisissantes, voire ahurissantes pour un lecteur non averti. Citons en particulier le texte d'Ernest Wamba-dia-Wamba sur l'église Bundu dia Kongo du Congo, ou encore les pratiques des Arathi du Kenya décrites par Francis Kimani Githieya, en passant par le Marian Faith Healing Ministry de Tanzanie étudié par Christopher Comoro et John Sivalon. Dans cette rubrique, on pourrait aussi évoquer l'église d'Alice Lakwena en Ouganda, sur laquelle on a beaucoup écrit et qui n'est que brièvement mentionnée ici. D'autres 
manifestations, moins extrêmes peut-être mais tout aussi importantes par les foules de croyants qu'elles attirent, témoignent de l'ampleur de ce foisonnement religieux en Afrique de l'Est. C'est le cas du Mouvement du renouveau (revival), phénomène de masse qui prend de cours les églises officielles: débordées par leur base, celles-ci tentent, tant bien que mal, de suivre le rythme. Si la plupart des témoignages rassemblés ici portent sur la Tanzanie (huit textes sur quinze), l'aspect géographique n'est pas le seul pertinent, car l'intensité de ces manifestations religieuses transcende incontestablement les frontières nationales.

3 Le deuxième objectif était de donner du christianisme africain une vision de l'intérieur. Les éditeurs entendent en effet se démarquer des études précédentes, dont ils dénoncent l'ethnocentrisme occidental, combler quelques lacunes (par exemple, insister sur le rôle déterminant des évangélistes dans la propagation de la foi) et porter un regard nouveau sur les faits religieux africains. Loin de considérer la christianisation comme un facteur parmi d'autres de la colonisation en insistant sur les avantages pratiques (éducation, santé, vêtements, etc.) que pouvaient tirer les gens de leur conversion, les auteurs soulignent la dimension morale du processus. S'ils se sont convertis au christianisme de façon si massive, c'est parce qu'ils y voyaient le moyen de retrouver, après les catastrophes du début du siècle, le contrôle moral de leur existence ("to regain a moral control over their lives", selon la formule de Spear dans l'introduction; ; . 3).

4 Il est vrai que les Africains, sans pour autant l'exprimer ouvertement, n'ont pas manqué de remarquer que les missionnaires étaient parfois bien loin d'appliquer dans leur propre conduite les préceptes de l'Évangile qu'ils s'efforçaient de propager. C'est ce que note sans aménité David Sandgren à propos des Kamba du Kenya. L'idée d'égalité contenue dans le message du Christ, révolutionnaire à son époque, menait derechef à la critique du comportement des autorités, qu'elles soient religieuses ou administratives, et contribuait ainsi à la lutte pour l'indépendance ou contre la corruption.

Le souci, de James Giblin notamment, de se faire l'écho du point de vue des Africains l'amène à critiquer l'ouvrage de Jean et John Comaroff, of Revelation and Revolution ${ }^{1}$, qui porte sur l'Afrique du Sud et a fait date dans l'historiographie du christianisme africain. Ces auteurs, selon lui (p. 309), mettent trop l'accent sur l'apport du christianisme, oubliant ce qui pouvait exister avant. Sans doute la minceur des sources antérieures au colonialisme peut-elle conduire à une telle dérive. Giblin poursuit avec un témoignage très nuancé sur les motivations qui pouvaient conduire les nouveaux chrétiens, dans le Sud tanzanien, à refuser la polygamie. Il ne s'agissait pas seulement d'appliquer une nouvelle règle morale; les arguments invoqués traduisaient avant tout la volonté de respecter les codes moraux en vigueur dans la société indigène. Les dilemmes auxquels ces nouveaux chrétiens étaient confrontés pouvaient être fort complexes, l'adhésion à une religion nouvelle ne gommant pas d'emblée d'autres règles très fortes telles que le respect des parents ou le souci de s'assurer une descendance.

6 Enfin, troisième point majeur : la diversité des situations rencontrées. Cette diversité tient, pour une part, à celle des missions venues s'installer sur le terrain, chacune apportant sa propre culture, ses propres manières de faire, et, bien souvent, son désir de l'emporter sur la voisine, perçue comme une rivale, donnant ainsi aux Africains le spectacle de ce que l'on pourrait qualifier de «tribalisme des missionnaires». Ces missions se sont ainsi trouvées confrontées sur le terrain à des situations et à des cultures indigènes extrêmement différentes, à tel point qu'elles sont loin d'avoir 
rencontré partout, loin s'en faut, le même succès. Souvent, d'ailleurs, le succès n'a pris corps qu'après une longue période de latence, quand les évangélistes africains ont pris le relais de la diffusion du message chrétien.

7 Ce livre contient ainsi quelques "success stories ", comme celle du catholicisme chez les Fipa racontée par Kathleen Smythe. Le succès est ici attribué, dans une large mesure, d'une part à la bienveillance des rois locaux, qui furent les premiers convertis, d'autre part à une certaine convergence religio-culturelle entre le catholicisme et la société fipa, restée à l'écart de l'impact colonial. Dans d'autres cas au contraire, comme celui de l'Africa Inland Mission chez les Maasai, décrit par Richard Waller, c'est un échec absolu qui s'observe, échec dont les causes sont implacablement retracées.

8 L'histoire de la christianisation de l'Afrique de l'Est offre donc des tableaux contrastés. À cette première source de diversité s'ajouta par la suite celle des conditions de la prise de contrôle, par les Africains, de leurs églises, thème que l'ouvrage aborde également. Or le nombre d'églises indépendantes qui ont vu le jour en Afrique de l'Est est saisissant. On compte au seul Kenya, d'après Gregory Maddox (p. 33), plus de trois cents églises indépendantes! L'ouvrage en mentionne quelques exemples, des plus orthodoxes aux plus extravagantes.

9 Ce livre apporte donc, sur un sujet aussi vaste que complexe, un panorama diversifié et judicieusement choisi. En bon outil de travail, il comporte une carte de situation des ethnies, un index et une excellente bibliographie.

\section{AUTEUR}

\section{CATHERINE BAROIN}

CNRS, Laboratoire de recherche sur l'Afrique, Maison René-Ginouvès, Nanterre. 